Physica 103B (1981) 315-323

(c) North-Holland Publishing Company

\title{
CONDUCTIVITY MEASUREMENTS WITH TRAPEZOIDAL A.C. MAGNETIC FIELDS
}

\author{
E. A. GIJSBERTSE and L. J. M. van de KLUNDERT \\ Twente University of Technology, Department of Applied Physics, P.O. Box 217, 7500 AE Enschede, The Netherlands
}

Received 4 August 1980

The inductive response of normal metals to trapezoidal a.c. fields is studied theoretically as well as experimentally. A method is presented to determine the conductivity and its magnetic induction dependence from the decay of the induced voltage during periods of constant field. The correctness of the analysis is confirmed by the close agreement between the complete experimental and theoretical voltage waveforms.

\section{Introduction}

Inductive techniques are a well known method for the measurement of the conductivity of metals. The main advantage of these techniques above the conventional four-probe method is that the experiment is essentially non-destructive. Furthermore, the method is more accurate for metals with a high conductivity. In principle two different techniques have been proposed: the decay curve method $[1-4]$ and the sinewave a.c. method [5]. In this a.c. method the conductivity is determined from the susceptibility of the sample in an alternating magnetic field of constant frequency. A careful analysis of this method, taking account of the finite dimensions of sample as well as coil system has been given by Rosenthal et al. [6]. In the decay curve technique the conductivity of the sample is extracted from the time constant of the decay curve of the induced voltage due to a sudden variation of a d.c. magnetic field. A difficulty with the latter method is that it is not possible to generate a perfect step in the magnetic field. A comparison between both techniques has been made by Wejgaard and Tomar [7].

The difficulty mentioned above may be avoided by making use of trapezoidally varying a.c. fields. The response of normal metals to a trapezoidal a.c. field has already been discussed in a previous paper [8]. It is the aim of this paper to evaluate this analysis further and to present a method, similar to the decay curve technique, which allows an accurate determination of the conductivity $\sigma$ even in the case of a dependence on magnetic induction. In a similar way the method can be used for the measurement of the flux flow conductivity $\sigma_{\mathrm{ff}}$ in type II superconductors or for a distinction between matrix losses and hysteresis losses in the superconducting filaments of multi-filament superconductors. The use of trapezoidal a.c. fields also provides a powerful technique for the determination of the bulk critical current density and the surface currents in type II superconductors $[9,10]$. This gives the possibility to determine the three contributions, i.e. bulk currents, surface currents and flux flow to the a.c. losses in superconductors separately.

In section 2 the response of normal metals (slabs and cylinders) in trapezoidal a.c. fields will be discussed while in section 3 the experimental results obtained on a Sn-sample are presented. From these results the conductivity of the sample as a function of magnetic induction has been determined. Finally, in section 4 , these experimental results will be compared with the theory.

\section{Theoretical analysis}

\subsection{General solutions}

The behaviour of an infinitely long non-magnetic cylinder of unit radius in an alternating magnetic field 
parallel to its axis can be described in terms of Maxwell's equations: $\operatorname{curl} \boldsymbol{E}=-\partial \boldsymbol{B} / \partial t$ and $\operatorname{curl} \boldsymbol{B}$ $=\mu_{0} j$. Under isothermal conditions the relation between $j$ and $E$ is given by Ohm's law $j=\sigma E, \sigma$ being the electrical conductivity which may depend on the local magnetic ind uction $\boldsymbol{B}$. In cylindrical coordinates these equations may be combined to:

$\frac{\partial^{2} B}{\partial r^{2}}+\frac{1}{r} \frac{\partial B}{\partial r}=\mu_{0} \sigma(B) \frac{\partial B}{\partial t}$.

Since only the $B_{z}$ and the $E_{\phi}$ components remain in this geometry, these subscripts have been omitted for reasons of briefness. It is assumed here that the cylinder is isotropic and that mean free path effects may be neglected. The cylinder is subjected to a trapezoidally varying magnetic field $B_{\mathrm{a}}(t)$ which has a constant value $B_{0}$ during some time interval for $t \leqslant 0$. During this interval all previously induced currents have disappeared. The boundary conditions now read:

$B(r, 0)=B_{\mathrm{a}}(0)=B_{0}, \quad 0 \leqslant r \leqslant 1 ;$

$\frac{\partial B}{\partial r}(0, t)=0, \quad$ for all $t$

$B(1, t)=B_{\mathrm{a}}(t)=B_{0}+\alpha t, \quad t \geqslant 0$.

In general, numercial methods have to be used for the solution of this type of equations. However, when the variations of the induction $B(r, t)$ inside the sample due to the induced currents are sufficiently small, the conductivity $\sigma(B)$ may be assumed to depend on the external induction only: $\sigma=\sigma\left(B_{\mathrm{a}}\right)$. In this case an analytical solution can be obtained with the help of finite Hankel transforms [11]. These are defined as:

$\vec{f}(p)=\int_{0}^{1} f(r) r J_{0}(p r) \mathrm{d} r$

where $p$ is any positive root of $J_{0}(p)=0$. The symbols $J_{0}(p r)$ and $J_{1}(p r)$ indicate the Bessels functions of zeroth and first order, respectively. The inverse transform reads:

$f(r)=2 \sum_{p} \bar{f}(p) J_{0}(p r) / J_{1}^{2}(p)$
With $B_{\mathrm{a}}$ as independent variable instead of $t$, eq. (1) transforms into:

$\frac{\mathrm{d} \vec{B}}{\mathrm{~d} B_{\mathrm{a}}}=\frac{p^{2}}{\mu_{0} \alpha \sigma\left(B_{\mathrm{a}}\right)}\left(-\bar{B}+B_{\mathrm{a}} J_{1}(p) / p\right)$,

with the boundary condition:

$\bar{B}\left(p, B_{0}\right)=\vec{B}_{0}=B_{0} J_{1}(p) / p$.

In the transform of (2a) into (6), the fact that $J_{1}(p) / p$ is the transform of unity, has been used. The solution of (5) is:

$\bar{B}\left(p, B_{\mathrm{a}}\right)=\left\{B_{\mathrm{a}}-I_{p}\left(B_{\mathrm{a}}\right)\right\} \cdot J_{1}(p) / p$,

with

$I_{p}\left(B_{\mathrm{a}}\right)=\mathrm{e}^{-g\left(B_{\mathrm{a}}\right)} \int_{B_{0}}^{B_{\mathrm{a}}} \mathrm{e}^{g(b)} \mathrm{d} b$,

and

$g\left(B_{\mathrm{a}}\right)=\frac{p^{2}}{\mu_{0} \alpha} \int_{B_{0}}^{B_{\mathrm{a}}} \frac{1}{\sigma(b)} \mathrm{d} b$.

The inverse transform now yields:

$B\left(r, B_{\mathrm{a}}\right)=B_{\mathrm{a}}-2 \sum_{p} p^{-1} I_{p}\left(B_{\mathrm{a}}\right) J_{0}(p r) / J_{1}(p)$,

and the average value of $B$ inside the sample is given by:

$B_{\mathrm{av}}\left(B_{\mathrm{a}}\right)=2 \int_{0}^{1} r B\left(r, B_{\mathrm{a}}\right) \mathrm{d} r=B_{\mathrm{a}}-4 \sum_{p} p^{-2} I_{p}\left(B_{\mathrm{a}}\right)$

In the case of a cylinder of radius $R$ eq. (9) must be replaced by:

$g\left(B_{\mathrm{a}}\right)=\frac{p^{2}}{\mu_{0} \alpha R^{2}} \int_{B_{0}}^{B_{\mathrm{a}}} \frac{1}{\sigma(b)} \mathrm{d} b$.

In this case the voltage induced in a pick-up coil around 
the sample can be determined from (11) according to $E=-\mathrm{d} \phi / \mathrm{d} t:$

$E\left(B_{\mathrm{a}}\right)=-\frac{2}{\mu_{0} \sigma\left(B_{\mathrm{a}}\right) R^{2}} \sum_{p} I_{p}\left(B_{\mathrm{a}}\right)$

The same result, of course, is obtained by combining Maxwell's equation $E=(\partial B / \partial r) / \mu_{0} \sigma\left(B_{\mathrm{a}}\right)$ and $(10)$. It can be estimated from (8) and (12) that for values of $B_{\mathrm{a}}-B_{0} \gg \mu_{0} \alpha \sigma_{\max } R^{2} p_{1}^{-2}\left(\sigma_{\max }\right.$ is the maximum value of $\sigma\left(B_{\mathrm{a}}\right)$ in the relevant field range and $p_{1}$ $=2.4048$ is the first root of $\left.J_{0}(p)=0\right), I_{p}\left(B_{\mathrm{a}}\right)$ is independent of the starting value $B_{0}$. This means that, independent of $B_{0}$, the $E$ vs. $B_{\text {a }}$ curve asymptotically approaches a single envelope curve which only depends on the relation $\sigma\left(B_{\mathrm{a}}\right)$ and on $\alpha$.

When at $t=t_{1}$ the external induction is kept constant again at a value $B_{1}=B_{0}+\alpha t_{1}, E$ decays to zero. In this case eq. (1) has to be solved once more; this time, however, with a constant value of $\sigma: \sigma_{1}=\sigma\left(B_{1}\right)$. The boundary conditions are now given by:

$$
\begin{array}{ll}
B\left(r, t_{1}\right)=B_{1}(r), & 0 \leqslant r \leqslant 1, \\
\frac{\partial B}{\partial r}(0, t)=0, & \text { for all } t, \\
B(1, t)=B_{\mathrm{a}}\left(t_{1}\right)=B_{0}+\alpha t_{1}=B_{1}, & t \geqslant t_{1},
\end{array}
$$

where $B_{1}(r)$ is given by eq. (10) with $B_{\mathrm{a}}=B_{1}$. The solution of this equation for a cylinder of radius $R$ is:

$$
\begin{aligned}
& B(r, t)=B_{1}-2 \sum_{p} I_{p}\left(B_{1}\right) p^{-1} \mathrm{e}^{-\left(t-t_{1}\right) / \tau_{p}} \\
& \quad \times J_{0}(p r) / J_{1}(p)
\end{aligned}
$$

where $\bar{B}\left(p, B_{1}\right)$ is given by eq. (7) and $\tau_{p}=\mu_{0} \sigma_{1} R^{2} / p^{2}$. From (15) it follows:

$$
B_{\mathrm{av}}(t)=B_{1}-4 \sum_{p} p^{-2} I_{p}\left(B_{1}\right) \mathrm{e}^{-\left(t-t_{1}\right) / \tau_{p}}
$$

and

$$
E(t)=\frac{2}{\mu_{0} \sigma_{1} R^{2}} \sum_{p} I_{p}\left(B_{1}\right) \mathrm{e}^{-\left(t-t_{1}\right) / \tau_{p}}
$$

\subsection{Determination of $\sigma$}

The general solutions obtained in the preceding section can be calculated numerically for any analytical $\sigma\left(B_{\mathrm{a}}\right)$ relation and even for tabulated values of $\sigma$ and $B_{\mathrm{a}}$. A simple and very interesting case, however, is the one in which $\sigma=\sigma_{0}$ is a constant (i.e. the absence of magnetoresistivity). This also is a good approximation when $\sigma$ varies only slightly with $B_{\mathrm{a}}$ within the field range of the a.c. field. In this case the equations (10), (11) and (13) reduce to (with $\tau_{p}=\mu_{0} \sigma_{0} R^{2} / p^{2}$ ):

$$
\begin{aligned}
& B(r, t)=\alpha\left\{t-2 \mu_{0} \sigma_{0} R^{2} \sum_{p} p^{-3}\left(1-\mathrm{e}^{-t / \tau_{p}}\right)\right. \\
& \left.\times J_{0}(p r) / J_{1}(p)\right\}, \\
& B_{\mathrm{av}}(t)=\alpha\left\{t-4 \mu_{0} \sigma_{0} R^{2} \sum_{p} p^{-4}\left(1-\mathrm{e}^{\left.-t / \tau_{p}\right)}\right\},\right. \\
& E(t)=-\frac{1}{2} \alpha\left\{1-4 \sum_{p} p^{-2} \mathrm{e}^{-t / \tau_{p}}\right\} .
\end{aligned}
$$

For large values of $t$ the value of $E(t)=-\frac{1}{2} \alpha$ becomes independent of $B_{\mathrm{a}}$. In this case (18) reduces to:

$B(r, t)=B_{\mathrm{a}}(t)-\frac{1}{4}\left(1-r^{2}\right) \alpha \mu_{0} \sigma_{0} R^{2}$,

showing the parabolic induction distribution inside the sample. Furthermore, in the limit for large $t$, eq. (19) reduces to

$\Delta B=\lim _{t \rightarrow \infty}\left\{B_{\mathrm{av}}(t)-B_{\mathrm{a}}(t)\right\}=(1 / 8) \alpha \mu_{0} \sigma_{0} R^{2}$.

In the derivation of (22) the relation $\Sigma_{p} p^{-4}=1 / 32$ has been used. Some other useful relations are $\Sigma_{p} p^{-2}$ $=1 / 4$ and $\Sigma_{p} p^{-6}=1 / 192$ [12]. When the external field is constant again, $E$ decays to zero according to:

$E(t)=-2 \alpha \sum_{p} p^{-2} \mathrm{e}^{-\left(t-t_{1}\right) / \tau_{p}}$

which is essentially the same as eq. (20).

From the foregoing it follows that there are two methods for the determination of $\sigma$ from experiments with trapezoidal a.c. fields. First of all eq. (22) may be used. In that case $\sigma$ is determined from the increase of internal induction after the external field is kept con- 
stant provided that the parabolic flux distribution has already been established. This very simple method, however, can be used only when $\sigma$ has a constant value; otherwise no simple relation between $\Delta B$ and $\sigma$ exists (see eq. (11)). In the latter case the second method has to be used. It can be seen from equation (17) that the $E$ vs. $t$ curve consists of a summation of exponential decay curves with different time constants $\tau_{p}=\mu_{0} \sigma_{1}$ $R^{2} / p^{2}$. For large values of $t$ only one of these terms remains: $\tau_{p_{1}}=\mu_{0} \sigma_{1} R^{2} / p_{1}^{2}$. The conductivity $\sigma_{1}$ $=\sigma\left(B_{1}\right)$ now can be determined by measuring the time constant $\tau_{p}$, in the tail of the decay curve. The relation $\sigma\left(B_{\mathrm{a}}\right)$ can be measured by repeating this experiment for various values of $B_{1}$.

It should be mentioned that $\tau_{p_{1}}$ increases with increasing $\sigma$ and with increasing cylinder radius $R$; as a consequence, the accuracy of the determination of $\sigma$ increases also. This is in contrast with the four-probe technique which measures the resistance of the cylinder. The latter decreases with increasing conductivity and increasing radius. So, for the measurement of a small conductivity in thin cylinders or slabs a fourprobe technique is preferable while in the case of large cylinders with a high conductivity the decay curve technique with either step-function like or trapezoidal a.c. fields are more accurate.

The derivation given so far has been restricted to a cylindrical geometry. In the case of a slab an analogous derivation can be given with the distinction that in this case finite Fourier transforms [11] have to be used; the time constants $\tau_{p}$ are replaced by $\tau_{k}$ $=4 \mu_{0} \sigma_{1} d^{2} /(k \pi)^{2}$ with $k=1,3,5$ etc. $(d$ is half the width of the slab) [8]. In the same paper also the influence of a not perfect trapezoidal waveform of the a.c. field has been discussed (coil effects). As was already mentioned before, perfect step-function field variations cannot be generated. In the special case of thermal instabilities in superconductors (so called flux jumps), however, an almost step-wise variation of the magnetic induction inside the sample together with a phase transition from the superconducting to the normal state has been observed [13]. In this case the normal conductivity of the sample can also be determined from the tail of the induced voltage peak.

\subsection{Numerical results}

Although the approximation $\sigma\left(B_{\mathrm{a}}\right)=\sigma_{0}$ is a good one for metals in which $\sigma$ depends only weakly on $B$, like copper, it is not sufficient for metals which exhibit a strong magneto-resistive effect like, for instance, tin or for superconductors in which flux flow effects have to be considered. The latter behaviour usually is described by a flux flow conductivity $\sigma_{\mathrm{ff}}=\sigma_{\mathrm{n}} B_{\mathrm{c}_{2}}(T) / B ;\left(\sigma_{\mathrm{n}}\right.$ denotes the conductivity in the normal state). According to Meaden [14] no general formula can be given for the transversal magneto-resistivity of metals. In the low field region a conductivity proportional to $B^{2}$ seems to apply while a $1 / B$-relation is a better approximation in the high field region. So in normal metals, as well as in superconductors, large variations of $\sigma$ with $B$ may occur. Therefore it is necessary to introduce a relation $\sigma\left(B_{\mathrm{a}}\right)$ in the solutions of section 2.1 .

In general this means that the integrals in eqs. (8) and (9) have to be evaluated numerically. Only in some special cases analytical solutions can be obtained: a discontinuous jump in $\sigma$ from one value to another and a linear variation of $\sigma$ with $B_{\mathrm{a}}[8]$. The latter case is of special interest as can be seen from the expression for the induced voltage in the limit for large values of $t$ :

$$
\lim _{t \rightarrow \infty} E=-\frac{1}{2} \alpha\left(1-\frac{1}{8} \alpha \mu_{0} \frac{\mathrm{d} \sigma}{\mathrm{d} B_{\mathrm{a}}}\right) .
$$

Equation (24) shows that the final value of $E$ differs from the value $-\frac{1}{2} \alpha$ for $\alpha\left(B_{\mathrm{a}}\right)=\sigma_{0}$ by a term proportional to $\mathrm{d} \sigma / \mathrm{d} B_{\mathrm{a}}$. It also can be seen from this expression that the influence of $\sigma\left(B_{\mathrm{a}}\right)$ disappears in the quasi-static limit $(\alpha \rightarrow 0)$.

A more realistic dependence of $\sigma$ on $B_{\mathrm{a}}$, appropriate for tin, is given by the relation [15]:

$$
\sigma=\sigma_{0} \frac{1+a B_{\mathrm{a}}^{2}}{1+b B_{\mathrm{a}}^{2}} .
$$

From experiments with a d.c. four-probe technique performed on a tin rod, the following values of the constants $\sigma_{0}, a$ and $b$ have been derived for the field range $B_{\mathrm{a}}<200 \mathrm{mT}$ :

$$
\sigma_{0}=0.932 \times 10^{10}(\Omega \mathrm{m})^{-1},
$$


$a=0.5 \times 10^{2} \mathrm{~T}^{-2}$

$b=0.8 \times 10^{3} \mathrm{~T}^{-2}$,

At low fields formula (25) exhibits the $B^{2}$-dependence mentioned before; in the high field limit (25) is no longer a good approximation of the experimental results. Although the constants for the sample described in the next section are somewhat different, the above $\sigma\left(B_{\mathrm{a}}\right)$ relation has been used in the calculations since it gives a more pronounced view of the characteristic features. A plot of $\sigma$ as a function of $B_{\mathrm{a}}$ is given in fig. 1. The introduction of eq. (25) for $\sigma\left(B_{\mathrm{a}}\right)$ allows an analytic integration of $g\left(B_{\mathrm{a}}\right) ; I_{p}$, however, has to be evaluated numerically. For the calculations a large number of terms $(n=50)$ has to be taken into account for the summation of $I_{p}$ to obtain sufficient accuracy.

A plot of some typical calculated $E$ vs. $B_{\text {a }}$ curves for different values of the starting field $B_{0}$ is given in fig. $2 \mathrm{a}$. It can be seen that for sufficiently large $\left(B_{\mathrm{a}}-B_{0}\right)$ values each curve coincides with the same envelope curve (see section 2.1.). For increasing applied field, the distance between this curve and the line $-E / \alpha=0.5$ first increases until a value of $B_{\mathrm{a}}$ slightly below 0 where the envelope curve has a minimum. This is in accordance with the result in eq. (24) since $\mathrm{d} \sigma / \mathrm{d} B_{\mathrm{a}}$ first increases and then has a maximum in the same field region. For positive values of $B_{\mathrm{a}}$, the envelope curve passes through $-E / \alpha=0.5$ as a consequence of the

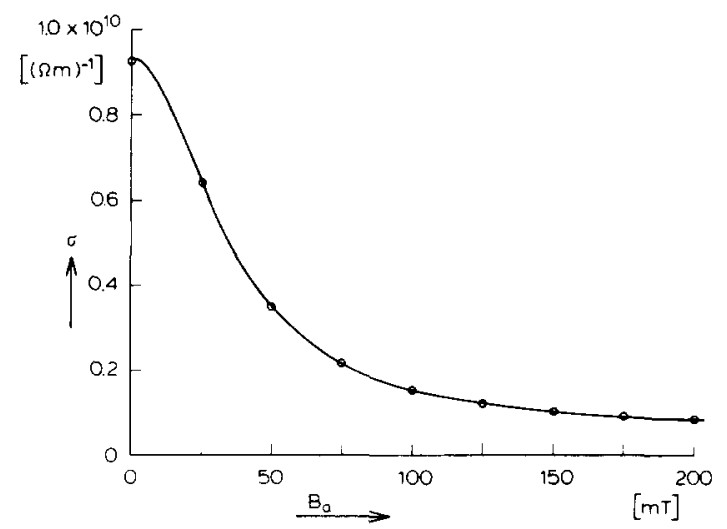

Fig. 1. The conductivity $\sigma$ as a function of $B_{\mathrm{a}}$ according to equations (25) and (26). Also the values determined from the calculated decay curves are indicated. change in sign of $\mathrm{d} \sigma / \mathrm{d} B_{\mathrm{a}}$. Although a relation between the envelope en $\mathrm{d} \sigma / \mathrm{d} B_{\mathrm{a}}$ definitely exists, it is not possible to determine $\sigma\left(B_{\mathrm{a}}\right)$ from this envelope curve. In fig. $2 \mathrm{~b}$ some calculated decay curves for different values of $B_{\mathrm{a}}$ are given. A few $E$ vs. $B_{\mathrm{a}}$ curves for different values of $\alpha=\mathrm{d} B_{\mathrm{a}} / \mathrm{d} t$ are shown in fig. 3 which illustrates that the complete observed behaviour, including the envelope curve, not only depends on the $\sigma\left(B_{\mathrm{a}}\right)$ relation but also on $\alpha$. In fact it is the quantity $\mathrm{d} \sigma / \mathrm{d} t=\alpha \cdot \mathrm{d} \sigma / \mathrm{d} B_{\mathrm{a}}$ which plays a dominant role (see eq. (22)). In the quasi-static approximation $(\alpha \rightarrow 0)$ the influence of $\sigma\left(B_{\mathrm{a}}\right)$ disappears.
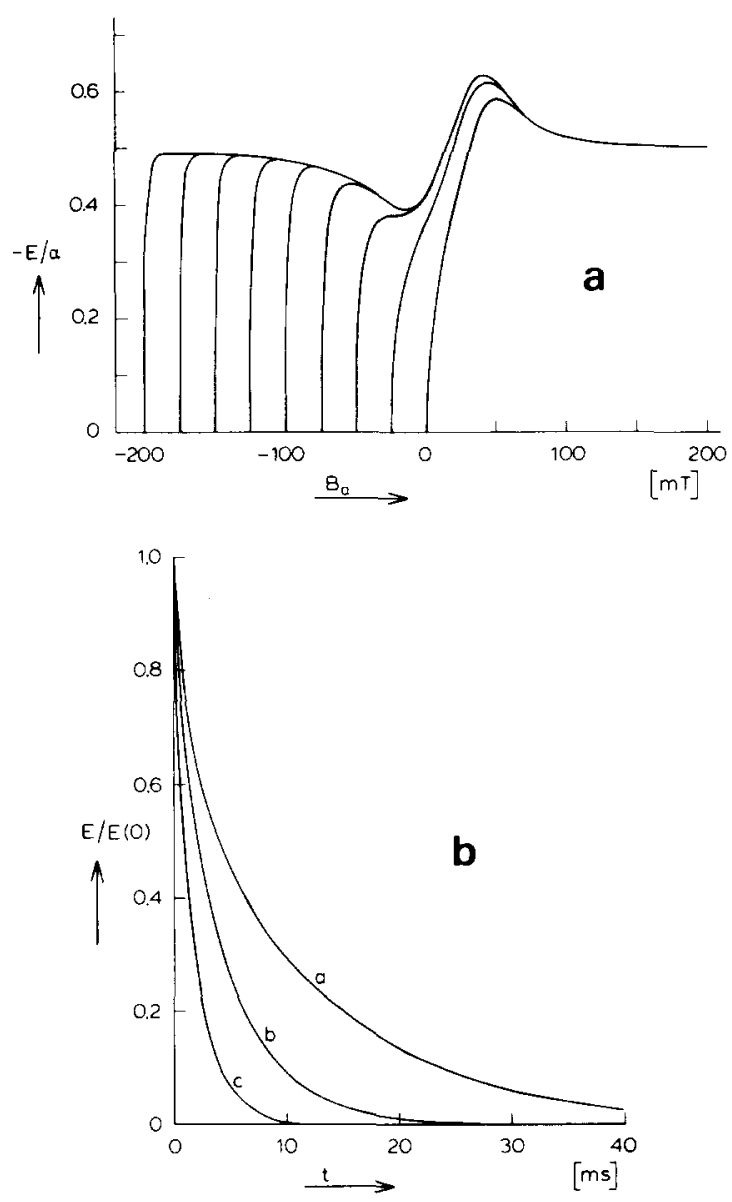

Fig. 2. (a) Calculated $E / \alpha$ vs. $B_{\mathrm{a}}$ curves for various values of the static field $B_{0}$. The $\sigma\left(B_{\mathrm{a}}\right)$ relation is given by (25) and (26). $\left(-100 \leqslant B_{0} \leqslant 100 \mathrm{mT} ; b_{0}=100 \mathrm{mT} ; \mathrm{d} B_{\mathrm{a}} / \mathrm{d} t=2 \mathrm{~T} / \mathrm{s}\right.$; $R=2.5 \mathrm{~mm}$ ). (b) Corresponding decay curves for three values of $B_{\mathrm{a}}:(\mathrm{a}): B_{\mathrm{a}}=0 \mathrm{mT}$; (b) $50 \mathrm{mT}$ and (c) $100 \mathrm{mT}$. 


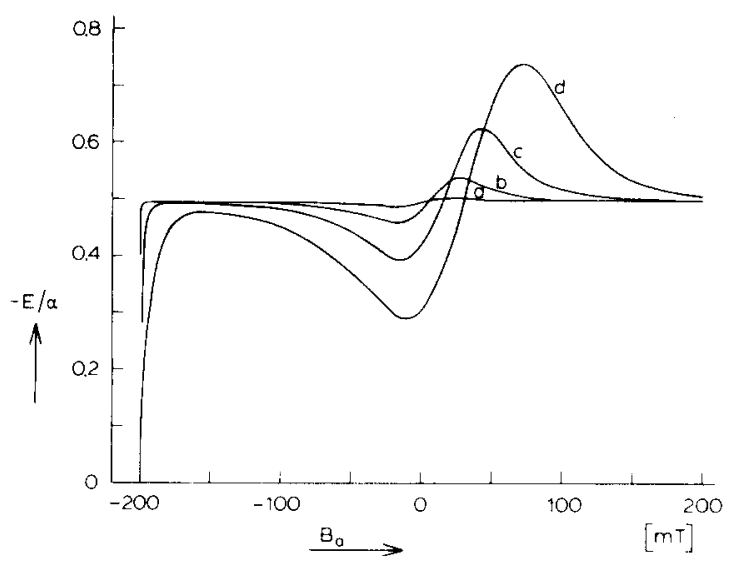

Fig. 3. Calculated $E / \alpha$ vs. $B_{\text {a }}$ curves for different values of $\alpha$ : (a) $\alpha=0.1 \mathrm{~T} / \mathrm{s}$; (b) $0.5 \mathrm{~T} / \mathrm{s}$; (c) $2 \mathrm{~T} / \mathrm{s}$ and (d) $8 \mathrm{~T} / \mathrm{s}$. $\left(B_{0}\right.$ $=0 \mathrm{mT} ; b_{0}=100 \mathrm{mT}$ and $R=2.5 \mathrm{~mm}$ ).

When $\Delta B=B_{\mathrm{a}}-B_{\mathrm{av}}$ is plotted vs. the applied inductions $B_{\mathrm{a}}$, a completely asymmetric picture arises, illustrating that eq. (22) is not valid in this case. A plot of $\Delta B$ vs. the average internal induction looks much more symmetric (see fig. 4 ) but also in that case no simple relation with $\sigma\left(B_{\mathrm{a}}\right)$ can be given. So, as expected, it is not possible to determine $\sigma$ from $\Delta B$. The only way for the determination of $\sigma$ therefore is to measure the time constant $\tau_{p_{1}}$ in the tail of the decay curve. This operation which has been used to determine the time constant of the experimental

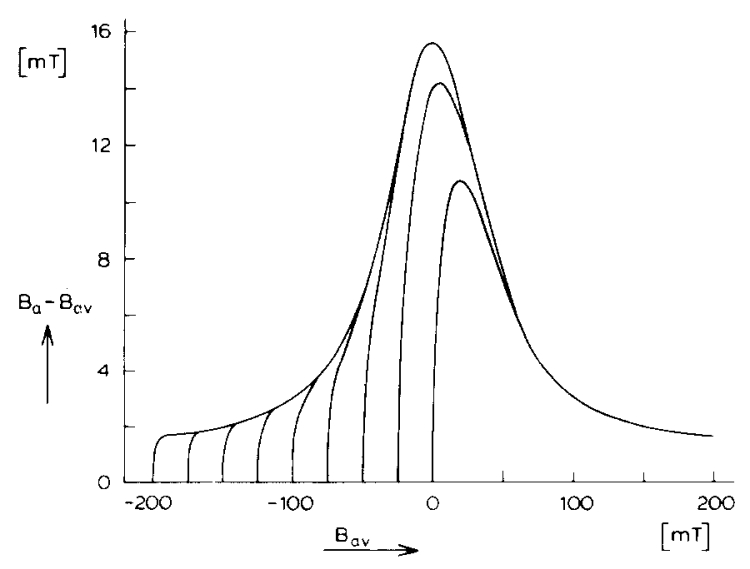

Fig. 4. $B_{\mathrm{a}}-B_{\mathrm{av}}$ as a function of $B_{\mathrm{av}}$ for various values of $B_{0}$ (see fig. 2). curves, also has been performed on the calculated decay curves. For these calculations the part of the decay curve between $E / E(0)=0.5$ and $E / E(0)$ $=0.1$ has been used, where $E(0)$ is the value of $E$ at the beginning of the decay curve. The time constant has been obtained by means of a least square deviation fit. The results, which are also indicated in fig. 1 agree within $0.5 \%$ with the exact value and do not depend on $\alpha$.

\section{Experimental results}

\subsection{Measuring technique}

The experiments described in this section have been performed with an equipment which is usually used for measurements on type II superconductors. A detailed description of the experimental set up has already been given elsewhere [10]. The sample is placed in an a.c. field of perfect trapezoidal waveform. The length of the horizontal parts of the trapezium has been chosen sufficiently long to allow the decay curves to vanish. As criterion for correct compensation for the unfilled part of the pick-up coil, the absence of discontinuities in the signal has been used [10]. The induced voltage curves have been recorded with the help of a transient recorder and evaluated by means of a computer. The value of $\sigma$ has been determined from the time constant of the decay curves by means of a least square deviation fit as described in the preceding section.

The range of $\sigma$-values which can be measured is determined by the frequency characteristic of the whole equipment. In the lower limit for the time constant, the behaviour of the pick-up coils (cutoff frequency above $2 \mathrm{MHz}$ ) and the maximum sampling frequency of the transient recorder $(2 \mathrm{MHz})$ have to be taken into account. This brings the lower limit down to a few microseconds. In most practical situations some additional chopper amplifiers are necessary. This brings the smallest time constant which can be measured up to $10 \mu \mathrm{s}$. In the upper limit the maximum observable time constant is determined by the sensitivity of the measuring system. This is a consequence of the low induced volt ages associated with very slow field variations. With our equipment time constants up to 10 seconds can be measured 
reasonably. The range of $\sigma$-values which can be measured also depends on the dimensions of the sample. For a cylinder of radius $R=2.5 \mathrm{~mm}$ this range extends from $7 \times 10^{6}(\Omega \mathrm{m})^{-1}$ to $7 \times 10^{12}$ $(\Omega \mathrm{m})^{-1}$. For comparison it should be mentioned that for a determination of $\sigma$ in the vicinity of $10^{12}$ $(\Omega \mathrm{m})^{-1}$ with a four-probe technique on a cylinder of the same radius and a length of $10 \mathrm{~cm}$, an accurate measurement of a resistance of about $5 \times 10^{-9} \Omega$ is required. For a slab of width $0.3 \mathrm{~mm}$ the $\sigma$-range extends from $10^{8}(\Omega \mathrm{m})^{-1}$ to $10^{14}(\Omega \mathrm{m})^{-1}$.

\subsection{Results}

Experiments have been performed on a polycrystalline tin ellipsoid with axes of $30 \mathrm{~mm}$ and $5 \mathrm{~mm}$. The ellipsoidal shape was chosen in order to avoid hysteresis in superconducting transitions; for a discussion of these effects we refer to Girard et al. [16]. The fact that an ellipsoid has been used instead of a cylinder has no large influence on the results. The pickup coil has been wound around a middle section of the sample over a length of $10 \mathrm{~mm}$. Most experiments were done with a trapezoidal a.c. field $\left(b_{0}=97 \mathrm{mT}\right.$, $\mathrm{d} B_{\mathrm{a}} / \mathrm{d} t=2.25 \mathrm{~T} / \mathrm{s}$ ) with a length of the horizontal parts of $75 \mathrm{~ms}$. A series of $E$ vs. $B_{\mathrm{a}}$ curves for different values of the static field is shown in fig. 5a. These curves also coincide after sufficiently long time and the envelope curve exhibits the same features as the calculated curve in fig. $2 \mathrm{a}$ although less pronounced. This is a consequence of the fact that the field dependence of $\sigma$ is less. In fig. Sb some experimental decay curves are shown for various values of the applied field. The value of the time constant decreases with increasing external field, as expected.

From these time constants the value of $\sigma$ has been determined. When the $\sigma$-values are plotted vs. the appropriate $B_{\mathrm{a}}$-values, the symmetry axis, which is expected at $B_{\mathrm{a}}=0$ is shifted to the negative $B_{\mathrm{a}}$ side. This is a consequence of the fact that in the vicinity of $B_{\mathrm{a}}=0$ the decreasing part of the a.c. field has been used. In this case the external induction is smaller than the internal induction during the decay curve. From this result it follows that actually $\sigma$ has to be considered a function of internal induction $B$ instead of external induction $B_{\mathrm{a}}$. In the same way the symmetry axis is shifted to the positive side when $\sigma$ is plotted vs. $B_{\mathrm{av}}$, the average induction at the beginning of the decay
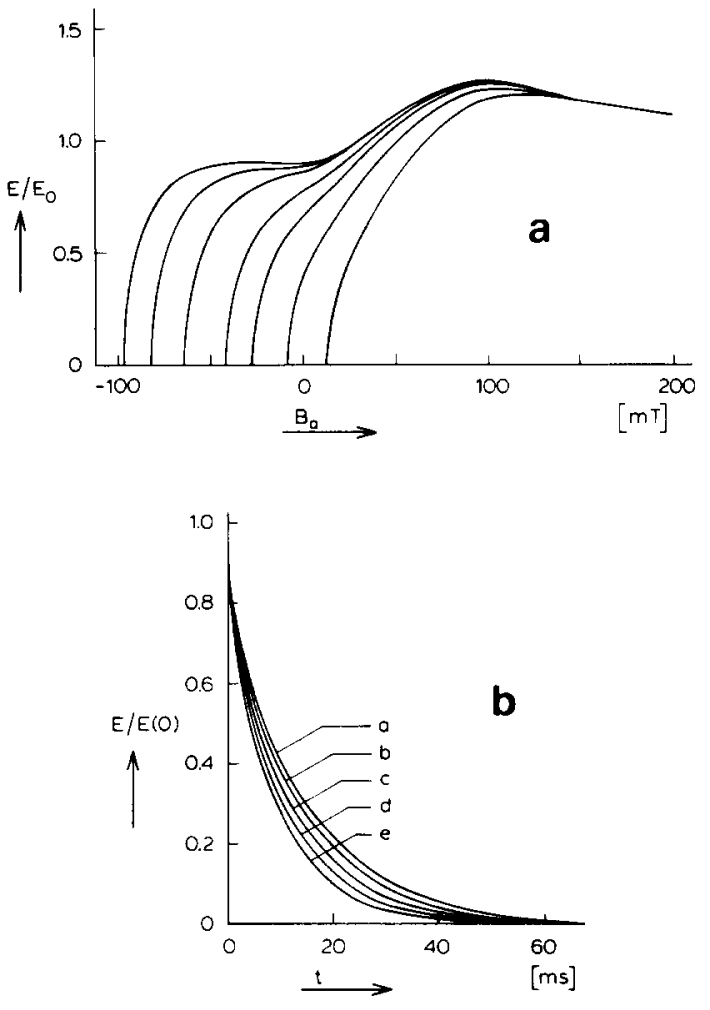

Fig. 5. (a) Experimental $E / E_{0}$ vs. $B_{\mathrm{a}}$ curve for tin for various values of $B_{0}\left(0<B_{0}<100 \mathrm{mT} ; b_{0}=97 \mathrm{mT} ; \mathrm{d} B_{\mathrm{a}} / \mathrm{d} t\right.$ $=2.25 \mathrm{~T} / \mathrm{s})(\mathrm{b})$ Corresponding experimental decay curves for different values of $B_{\mathrm{a}}$ : (a) $B_{\mathrm{a}}=30 \mathrm{mT}$; (b) $45 \mathrm{mT}$; (c) $67 \mathrm{mT}$; (d) $85 \mathrm{mT}$ and (e) $100 \mathrm{mT}$.

curve. A good compromise is obtained by plotting $\sigma$ vs. $B^{*}=\left(B_{\mathrm{a}}+B_{\mathrm{av}}\right) / 2$ which is a good approximation for the internal flux density during the decay curve. The results are given in fig. 6 for the decay curves measured at both the minimum and the maximum of the a.c. field and for different amplitudes. The results agree well.

Also some points for negative $B^{*}$ have been imaged with respect to $B^{*}=0$, giving good results. A reasonable analytic approximation of these results is given by eq. (25) with the constants:

$$
\begin{aligned}
& \sigma_{0}=1.32 \times 10^{10}(\Omega \mathrm{m})^{-1} \\
& a=0.20 \times 10^{2} \mathrm{~T}^{-2}, \\
& b=0.14 \times 10^{3} \mathrm{~T}^{-2} .
\end{aligned}
$$






Fig. 6. Experimental values of the conductivity $\sigma$ as a function of $B^{*}=\left(B_{\mathrm{a}}+B_{\mathrm{av}}\right) / 2$. Results for increasing as well as decreasing fields and obtained with different amplitudes of the a.c. field are given.

\section{Discussion}

In the preceding sections the behaviour of a normal metal in a trapezoidal a.c. magnetic field has been discussed extensively and a method has been presented to determine the conductivity of the metal for different values of the magnetic induction from the decay curves. A good criterion for the correctness of the obtained results is whether a numerical calculation according to section 2.3, making use of the experimental $\sigma$-values, produces a voltage waveform which corresponds to the experimentally observed curve. Although the conductivity has been determined from the tail of the decay during the constant part of the trapezium only, the whole induced waveform may be used as a criterion. In fig. 6 the conductivity has been plotted versus the average internal induction $B^{*}$ during the decay curve while a $\sigma\left(B_{\mathrm{a}}\right)$ relation is used in the calculation. This means that for a good comparison of theoretical and experimental results one has to introduce the experimental $\sigma\left(B_{\text {av }}\right)$ curve in the calculations rather than $\sigma\left(B_{\mathrm{a}}\right)$ or $\sigma\left(B^{*}\right)$. In increasing field this $\sigma\left(B_{\mathrm{av}}\right)$-value has its maximum when $B_{\mathrm{av}}=0$ i.e. at a positive value of $B_{\mathrm{a}}$. A consequence of this approximation is that one has two different $\sigma\left(B_{\mathrm{a}}\right)$ relations for increasing and decreasing field respectively. Since the actual $B_{\mathrm{av}}$-values during the whole field sweep are not known, the $B_{\mathrm{av}}$-values used in the calculations below have been obtained from the experimental envelope $B_{\mathrm{av}}-B_{\mathrm{a}}$-curve. A comparison between theoretical and experimental results is given in fig. $7 \mathrm{a}$. for the increasing part of the trapezium and in fig. $7 \mathrm{~b}$ for the constant time interval. Good agreement has been obtained. Only at the beginning of the $E$ vs. $B_{\text {a }}$ curve of fig. 7a some minor deviations are observed which can be ascribed to the fact that the $B_{\text {av }}$-values of the envelope curve have been used instead of the actual values. 

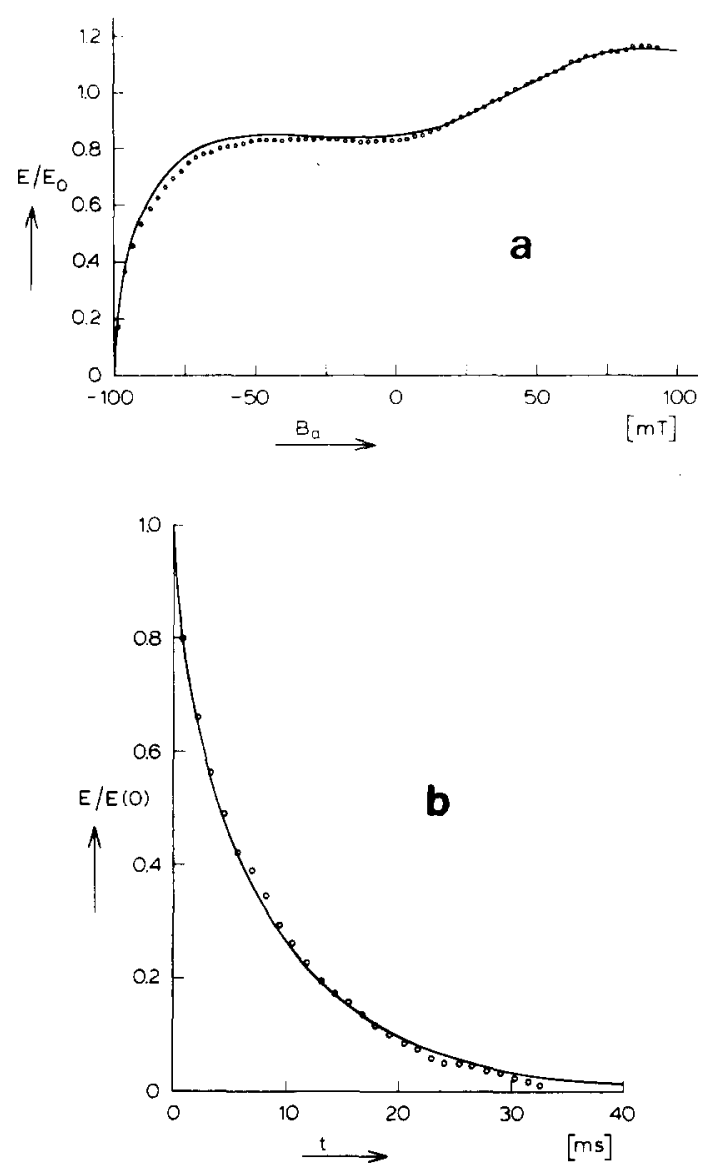

Fig. 7. (a) Comparison between theoretical and experimental waveform in increasing field. The solid line represents the theoretical curve $\left(B_{0}=0 \mathrm{mT} ; b_{0}=100 \mathrm{mT} ; \mathrm{d} B_{\mathrm{a}} / \mathrm{d} t=2.25 \mathrm{~T} / \mathrm{s}\right.$ and $R=2.5 \mathrm{~mm}$ ) while the points give the experimental results $\left(B_{0}=0 \mathrm{mT}, b_{0}=97 \mathrm{mT}, \mathrm{d} B_{\mathrm{a}} / \mathrm{d} t=2.25 \mathrm{~T} / \mathrm{s}\right.$ and $R=2.5 \mathrm{~mm}$ ). (b) Corresponding decay curve for $B_{\mathrm{a}}=100 \mathrm{mT}$.

The comparison given above shows that the behaviour of normal metals with conductivities upto at least $10^{10}(\Omega \mathrm{m})^{-1}$ can be described with a model in which the conductivity $\sigma$ is a function of applied induction only and does not depend on the position inside the sample. For a good interpretation of the results, however, it is necessary to take into account the value of the average induction inside the sample as well. The conductivity of these metals can be determined experimentally with great accuracy with the help of trapezoidal a.c. fields. For metals with a much larger conductivity or a much larger radius the assumption that $\sigma$ is independent of position may no longer hold and eq. (1) has to be solved numerically. In the case of a large conductivity also mean free path effects must be taken into account. For most metals, however, $\sigma$ is much lower, in which case the technique presented here provides accurate results with respect to the value of $\sigma$ and its dependence on magnetic induction. This conclusion also applies for the flux flow conductivity in type II superconductors which means that the use of trapezoidal a.c. magnetic fields provides a good method for the determination of the loss contribution of these effects.

\section{References}

[1] C. P. Bean, R. W. DeBlois and L. B. Nesbit, J. Appl. Phys. 30 (1959) 1976.

[2] A. F. Clark, V. A. Deason and R. L. Powell, Cryogenics 12 (1972) 35.

[3] R. C. Calarotti and M. Alfonzo, J. Appl. Phys. 43 (1972) 3040.

[4] R. C. Calarotti, J. Appl. Phys. 43 (1972) 3949.

[5] J. E. Zimmerman, Rev. Sci. Instrum. 32 (1961) 402.

[6] M. D. Rosenthal and B. W. Maxfield, Rev. Sci. Instrum. 46 (1975) 398.

[7] W. Wejgaard and V. S. Tomar, J. Phys. E7 (1974) 395.

[8] L. J. M. van de Klundert, H. P. van de Braak and E. A. Gijsbertse, Physica B90 (1977) 237.

[9] L. J. M. van de Klundert, E. A. Gijsbertse and H. P. van de Braak, Physica B94 (1978) 41.

[10] E. A. Gijsbertse, M. Caspari and L. J. M. van de Klundert, submitted to Cryogenics.

[11] C. J. Tranter, Integral Transforms (Methuen, London, 1966).

[12] P. le Grand, private communications.

[13] E. A. Gijsbertse, L. J. M. van de Klundert, M. L. D. van Rij and W. J. van Weezep, submitted to Cryogenics.

[14] G. T. Meaden, Electrical resistance of metals (Heywood Books, London, 1966).

[15] M. Kohler, Ann. Phys. 6 (1949) 18.

[16] J. P. Girard, E. Paumier and A. Fortini, J. de Phys. 32 (1971) 895 . 